\title{
The role of radiological protection experts in stakeholder involvement in the recovery phase of post-nuclear accident situations: Some lessons from the Fukushima-Daïchi NPP accident
}

\author{
Thierry Schneider ${ }^{1, *}$, Mélanie Maître ${ }^{1}$, Jacques Lochard ${ }^{2}$, Sylvie Charron ${ }^{3}$, Jean-François Lecomte ${ }^{3}$, \\ Ryoko Ando ${ }^{4}$, Yumiko Kanai ${ }^{5}$, Momo Kurihara ${ }^{6}$, Yujiro Kuroda ${ }^{7}$, Makoto Miyazaki ${ }^{7}$, Wataru Naito ${ }^{5}$, \\ Makiko Orita $^{2}$, Noboru Takamura ${ }^{2}$, Koichi Tanigawa ${ }^{7}$, Masaharu Tsubokura ${ }^{7}$ and Tetsuo Yasutaka ${ }^{5}$ \\ ${ }^{1}$ Nuclear Protection Evaluation Center (CEPN), 28 rue de la Redoute, 92260 Fontenay-aux-Roses, France. \\ 2 Nagasaki University, Atomic Bomb Disease Institute, Nagasaki, Japan. \\ 3 Radiation Protection and Nuclear Safety Institute (IRSN), Fontenay-aux-Roses cedex, France. \\ ${ }^{4}$ Ethos in Fukushima/ NPO Fukushima Dialogue, Fukushima, Japan. \\ ${ }^{5}$ National Institute of Advanced Industrial Science and Technology (AIST), Tsukuba, Japan. \\ ${ }^{6}$ National Institutes for Quantum and Radiological Science and Technology (QST), Chiba, Japan. \\ ${ }^{7}$ Fukushima Medical University (FMU), Fukushima City, Japan.
}

Received: 18 September 2019 / Accepted: 23 September 2019

\begin{abstract}
Feedback experiences from Fukushima and Chernobyl situations have clearly shown the importance of involving local stakeholders living in contaminated territories for the rehabilitation of their daily life. In this context, this paper aims to better address the role of radiological protection experts in the recovery phase of post-nuclear accident situation, in mainly relying on the analysis of local initiatives implemented in the Fukushima Prefecture following March 2011. In the first part, this paper highlights the various challenges faced by the population living in contaminated territories, i.e., rehabilitation of the living conditions, ensuring a long-term radiological monitoring, developing public health programs. In a second part, this paper discusses to which extent radiological protection experts can help local population to address these challenges, particularly through the implementation of co-expertise processes and the associated ethical issues and values they should embody. The last part of this paper particularly focuses on two current challenges at stake in the Fukushima Prefecture: the dissemination of the co-expertise process to all affected communities, as well as the sustainability of these approaches over time.
\end{abstract}

Keywords: radiological protection expert / local communities / co-expertise process / Fukushima / post-accident

\section{Introduction}

The Fukushima accident, as previously that of Chernobyl, highlighted the importance of the involvement of local stakeholders living in the contaminated areas especially for the management of the recovery phase for allowing them to take informed decisions as well as ensuring the effectiveness and sustainability of protective actions. The support of national and local authorities and radiological protection experts ${ }^{1}$ plays

\footnotetext{
*Corresponding author: thierry.schneider@cepn. asso.fr

${ }^{1}$ In this paper, radiological protection experts should be taken in a broad sense, that is all experts contributing to address the radiological consequences of a nuclear accident.
}

a key role to initiate and accompany this stakeholder involvement. Experience from Japan emphasizes the conditions and challenges for a fruitful, sustainable and respectful engagement of experts and their organizations.

The aim of this paper is in the first part to review some challenges in the recovery phase for local population, several years after the Fukushima accident, taking into account the lessons drawn on the past experiences from Belarus and Norway on stakeholder involvement (Liland and Skuterud, 2013; Lochard, 2013). The issues at stake are mainly to rehabilitate the living conditions, to ensure the long-term radiological monitoring and to develop public health programs. In the second part, the role of experts to accompany the local population for addressing these challenges is discussed particularly in relation with the co-expertise process. 


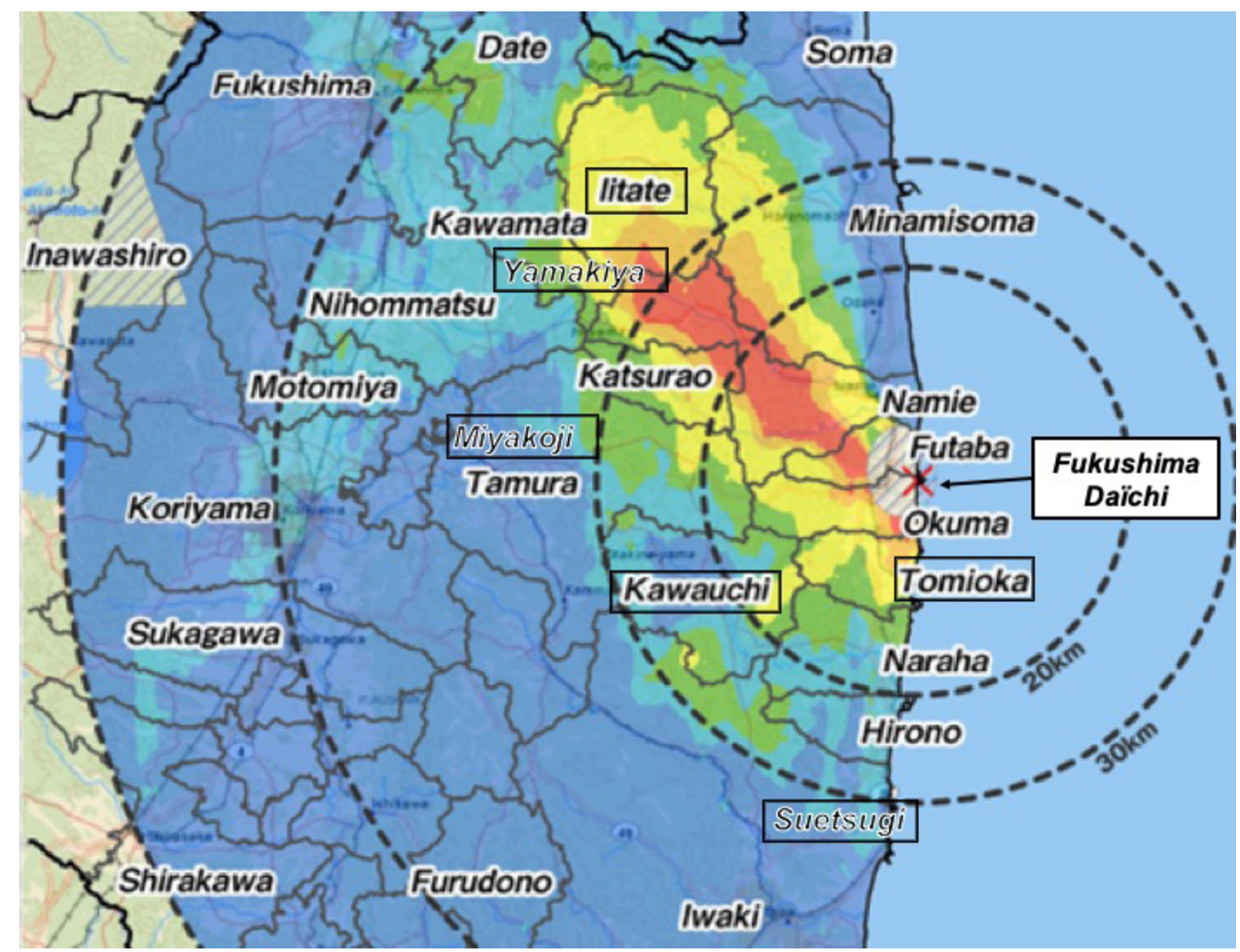

Figure 1. Location of the 6 initiatives of co-expertise detailed in this paper.

Finally, the dissemination of the co-expertise process to all affected communities, as well as the sustainability of the stakeholder involvement approaches are highlighted in the last part.

\section{Methodology}

The analysis presented in this paper mainly relies on a series of interviews of radiological protection experts and stakeholders directly involved in local initiatives for the recovery process in the Fukushima Prefecture. It also takes into account the main lessons of the Fukushima Dialogue meetings initiated by the International Commission on Radiological Protection (ICRP) (Ban, 2016; Lochard et al., 2019).

Since the autumn 2011, a collaboration has been progressively established between Japanese experts, local stakeholders involved in the recovery process and French experts from CEPN and IRSN through regular visits to local communities affected by the Fukushima accident, the participation together in various meetings as well as the development of joint analyses. This collaboration gradually allowed to better understand the role of radiological protection experts to accompany the implementation of radiological protective actions at the local level in the recovery phase of the accident and, more particularly, in the co-expertise process implemented in some communities. These findings have been regularly shared and discussed with a broader set of experts and stakeholders in Japan and in France over the last years (Gariel et al., 2018). Furthermore, in December 2017, a seminar, jointly organized by Nagasaki University, CEPN and IRSN, was held in Nagasaki to share the current experiences of stakeholder involvement with experts from Japan, Europe and international organizations, as well as professionals and leaders from Fukushima Prefecture. A particular emphasis was placed on better identifying and understanding the ethical issues associated with stakeholder involvement, risk communication and the development of the practical radiological protection culture in the recovery phase after a nuclear accident.

Several initiatives of co-expertise in the recovery phase of the Fukushima accident have been identified and reviewed in order to investigate the challenges and difficulties for radiological protection experts to engage themselves in such approaches:

- the initiative of the Suetsugi villagers with the support of the NPO "Ethos in Fukushima" and the involvement of Fukushima Medical University experts;

- the initiative of the municipality of the Kawauchi village with the support of Nagasaki University;

- the joint initiative of Tomioka city and Nagasaki university following the lifting of the evacuation order;

- the initiative of the municipality of Iitate village in the context of the preparation of the lifting of evacuation order with the support from Fukushima Medical University;

- the involvement of the residents of Miyakoji village in the measurements of their individual dose with the support of Fukushima Medical University;

- the initiative of citizens of Yamakiya village following the lifting of evacuation order with the support of AIST.

These initiatives, located on Figure 1, are briefly described in the Box 1. 


\section{Box 1. A summary presentation of the co-expertise initiatives having supported the present analysis}

The Suetsugi community

Launched in 2017, this project, led by Fukushima Medical University (FMU) in cooperation with the Japanese NGO called ETHOS in Fukushima, aims to create an Atlas retracing experience of the villagers of Suetsugi since the accident (Ando, 2016; ICRP, 2016). To this end, all the measurement data on the radiological situation have been collected, interviews with the inhabitants of the village were carried out, together with the support of Hiroshima University. A video ${ }^{2}$ and a document presenting the history of the approach of the inhabitants of the village of Suetsugi have been prepared. In addition to the historical background, the aim of this document is to identify the issues in terms of vigilance in relation to the radiological situation, the methods for disseminating the approach and the current and future challenges for the residents of the village.

Analyzing the radiological measurement data collected since 2011 contributes to highlight the evolution of the situation after the accident and potentially constitutes a database that could be exploited in the framework of a more global analysis of the radiological situation of the territories contaminated by the Fukushima accident.

Besides providing information on the radiological characterization of the village, developing the Atlas makes it possible to begin a work of memory for the inhabitants of the village and gives them a unique opportunity to be able to express their feelings compared to what they lived and with regard to their future. It should be noted that the project was designed to allow the production of a document and a video without having pre-established a method of dissemination. However, it appears that the existence of this project allows project owners and inhabitants to contact different organizations and experts at local, national and even international levels, engaging the inhabitants in a dynamic of sharing their experience.

\section{The Kawauchi village}

The involvement of Nagasaki University with Kawauchi village has evolved over time (Takamura et al., 2018). Following the accident, 10 food contamination monitoring devices were set up and distributed in the different areas of the village. Progressively, their number has been reduced to 3 , due to the decrease of requests from residents. Information is still provided to the residents and regular exchanges with the local authorities are organized on the evolution of the radiological situation of food and exposures. Dedicated workshops are organized with school students in Kawauchi village to develop their awareness on the local radiological situation and each year, a group of Kawauchi school students is visiting Nagasaki University.

\footnotetext{
${ }^{2}$ Regaining confidence after the Fukushima accident: the story of the Suetsugi Community, 2018. https://www.youtube.com/watch?v= L_ZhjixM6oM\&feature=youtu.be.
}

Nowadays, Nagasaki University relies on the approach on risk communication developed in Kawauchi village to ensure the training of students coming from Nagasaki University, FMU, as well as future teachers from Fukushima Prefecture.

These different activities contribute significantly to maintaining the vigilance for Kawauchi residents, reinforcing the sustainability of the strong partnership between the local community and Nagasaki University.

\section{The Tomioka town}

Neighboring city of Kawauchi, Tomioka is considered to be the twin city of Kawauchi. Therefore, right after the Fukushima accident and the first evacuation orders, residents of Tomioka evacuated to Kawauchi before evacuating further. The evacuation order of the town of Tomioka has been lifted in April 2017 on $85 \%$ of its territory. At the end of June 2019, only 1064 people out of $15,961^{3}$ live in Tomioka and most of them are elderly people or new workers of the Fukushima Nuclear Power Plant. Based on its experience in Kawauchi, Nagasaki University is involved in Tomioka, proposing and adapting the actions which were implemented in Kawauchi (Takamura et al., 2018). Therefore, group discussions on radiological risk are proposed to the residents of Tomioka. Similarly, home visits are organized by experts from the Nagasaki University, as well as measurements of the environment and the local products. Technical assistance is also ensured several times per week in the premises of the Municipality hall of Tomioka. Actions at the Tomioka school are also conducted by experts in order to raise awareness of young children about the radiological situation of their environment.

Nowadays in Tomioka, the major challenge is to make newcomers aware about the radiological situation of the city, and provide them radiological protection advises. Also, since residents returning to live in Tomioka are often isolated, experts try to ensure a link between all the community members.

\section{The Iitate village}

The emergency phase (especially evacuation) as well as medium and long-term upheavals in life resulting from the accident induced not only physical and mental health effects but also affected the social well-being of Iitate evacuees. For instance, the changes in the living conditions led to an increase in diabetes, cardio-vascular diseases, obesity (because of a lack of physical activities), stress, etc. Social factors such as the post-disaster losses of social support and social capital have affected the well-being of Iitate residents who were evacuated in temporary housing. In such situation, community of health workers from the village played a key role for the residents. They have been at the forefront of dialogues with the residents since the very early stage of the accident. Their role is to provide community members health-related advice, but it has become difficult in the nuclear accident aftermath (Kuroda et al., 2018a, 2018b). Together with a psychologist from

\footnotetext{
${ }^{3}$ www.tomioka-town.jp/soshiki/jumin/jumin/hinansya_ninzu/2594. html (in Japanese).
} 
Fukushima Medical University, they initiated various actions, especially, round-table dialogues in all 'Kasetsu' (temporary houses) and a nursery for young children from litate. These dialogues allow villagers, especially young mothers, to expose their concerns and worries such as promiscuity problems, anxieties with regards to potential health consequences for their children and food safety, etc.

\section{The Miyakoji village}

The Miyakoji village, located 20 to $30 \mathrm{~km}$ of the Fukushima power plant, was evacuated at the time of the accident. Due to the limited level of contamination of the area, the order of evacuation was lifted on April $1^{\text {st }}, 2014$. Several farmers have progressively restarted their activities, while others are still confronted to difficulties or have abandoned their activities. Social infrastructures, notably the school, have been reestablished allowing to restart the social life in the village. In June 2019, the number of residents living in Miyakoji was about $80 \%$ of the population before the accident ${ }^{4}$. In this context, it should be noted the key role of the support provided by radiological protection experts, notably from Fukushima Medical University, who set up a dialogue with local residents and provided them the means to measure their individual doses with the "D Shuttle" dosimeter (Chiyoda Technol, 2018). These measurements allowed the residents to assess their own situation taking into account the specific radiological situation in the village and contributed to the decision of the residents to allow the lifting of the evacuation order (Miyazaki, 2017).

\section{The Yamakiya village}

Yamakiya village was the only restricted area of Kawamata town. The evacuation order of the Yamakiya village has been lifted at the end of March 2017. In June 2019, only 363 people ${ }^{5}$ returned to Yamakiya out of about 1200 who lived in the village before the accident and most of them are elderly people.

The radiological characterization of the local environment and the establishment of protective actions have been performed with the experts from AIST, Chiba university and different research organizations and universities contributing to create the conditions for understanding the local environment (e.g. soil, water and forest) (Kurihara et al., 2018a, 2018b; Tsuji et al., 2014). However, after 2017, the interests of local people who have returned or wanted to return gradually evolve from environmental issues to social and economic issues such as the restart of agricultural production.

In this context, the experts from AIST and local population decided to organize an onsite interactive learning activity program named Yamakiya Gakko (Yamakiya School, held about 6-7times a year) that includes flower farm work assistance, wild plant survey and dialogue with local residents mobilizing various people from outside who contribute to the activities (Yasutaka et al., 2019). The network around Yamakiya

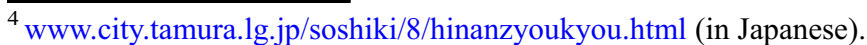

${ }^{5}$ https://www.town.kawamata.lg.jp/site/sinsai-saigai/yamakiyatiku kyojyuujyoukyou.html (in Japanese).
}

Gakko is now a very informal one, operating on a voluntary basis. Composed of about 100 people, this network includes:

- about $40 \%$ of researchers and their students from universities inside and outside of the Fukushima Prefecture;

- about $30 \%$ of researchers from research organizations;

- about $30 \%$ of people from private companies, officers of the local authority and government.

It should be noted that these volunteers help Yamakiya residents on their spare time and their involvement in the revitalization of this territory is quite personal. However, when these people come to participate in the activities, informal discussions take place between these volunteers and the local population. These exchanges address various issues, including not only information on the radiological situation of the territory and the dissemination of practical advice in radiological protection but also local population interest, e.g. latest knowledge of the flower cultivation, utilization of the forest, etc.

\section{What is at stake for people and communities living in affected territories?}

Several years after the accident, the main concern is focused on psycho-socio-economic issues in order to ensure the quality of the living conditions for people residing in affected territories. The radiological situation remains an important issue but the radiological characterization of the territories has already been established and the decisions for allowing or not people to live in the territories have been taken (see contamination map provided in Fig. 2). Therefore, it is no more the central issue, although there is a need to organize the long-term radiological monitoring. In addition, besides the possible radiation-induced health effects associated with exposure to ionizing radiation, there is a general concern on public health issues for the inhabitants in their daily life as well as for possible health effects on future generations.

\subsection{Rehabilitation of living conditions}

One of the first issues for the local communities following the accident concerns the capability to restart agriculture activities as well as the attractiveness of the affected areas for implementing new economic activities (Baudé et al., 2016). It appears to be a pre-requisite to envisage the possible future of the daily life in affected territories. However, as in any industrialized country, there is a trend in Japan itself for a desertification of the countryside and a decrease of the rural fabric, leading to the aging of the agricultural population. Since 2011, this process has been accelerated in the Fukushima Prefecture.

The main objective for local authorities is to re-create decent living conditions with reference to the previous situation before the accident and expecting to restore as fast as possible similar conditions for the population living in 


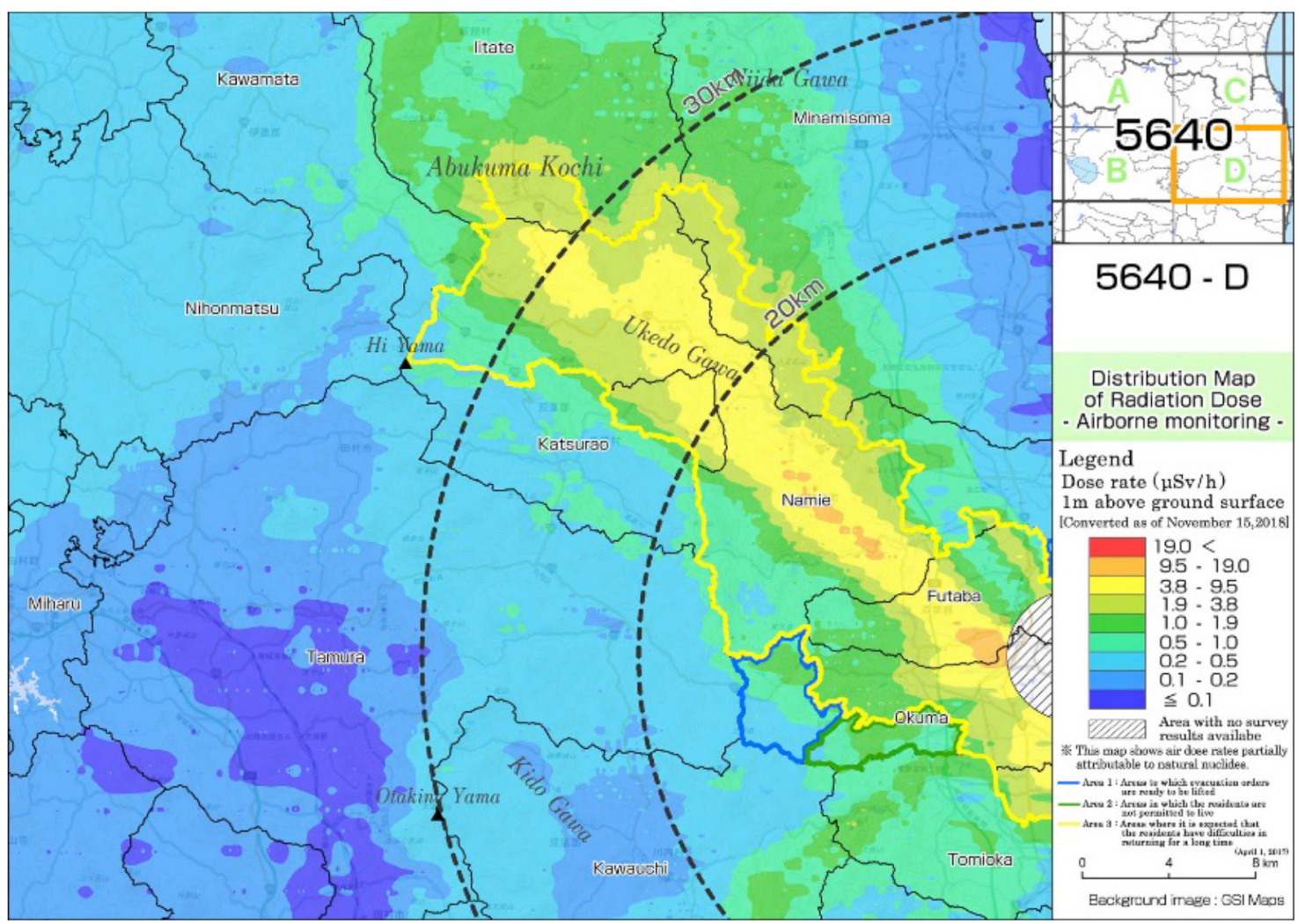

Figure 2. Ambient dose rates map within the Fukushima Prefecture, as of November 15th, 2018 (source: Geospatial information authority of Japan - Ministry of Land, Infrastructures, Transport and Tourism).

affected territories. Public investments are made to reconstruct or reinforce the public infrastructures related to transport (road, railway), education and healthcare. After the Fukushima accident, these infrastructures have been developed by local authorities with a long-term perspective having in mind a possible increase of residents, appearing sometimes disproportionate with regard to the current situation (ex. schools reopened in Kawauchi, Tomioka, Iitate).

Nevertheless, many obstacles and difficulties appear revealing the complexity and long-lasting consequences of the post-accident situations with regard to the daily life (ex. Yamakiya and Iitate with lack of manpower for farm activities). Residents living in affected areas frequently express their concern associated with the destruction of their local community: they have no more the same neighbors, their family life is not the same as before, there is no more the same number of children at school, some residents have a different job, and the commercial activities have significantly changed following the evacuation of some areas. In this context, people express a lack of places of dialogue as well as their difficulties to exchange on their concerns, experiences and expectations with other residents and with the local authorities (Kotoba, 2015).

In the evacuated territories arises the question of the degradation of the houses as well as the public and community places. The lack of human activity for several years has led to a gradual deterioration of the premises that creates a complex situation for residents expecting to return. In such a situation, the preservation of heritage for future generations remains a major concern for the local community.
It is worth to mention that, facing this situation, traditional practices, which have been interrupted after the accident, are now restored and seem to play a crucial role in the rehabilitation of living conditions of communities following the accident. These are festivals like in Suetsugi for example or traditional dances like in Futaba. Similarly, the natural heritage, the traditional economic or agricultural activities are all elements that mark the history of the communities and influence the restart of their economic and social activities (Lochard et al., 2019).

These specificities, however, constitute a difficulty for the central authorities, which are striving to propose a generic program in order to facilitate rapid recovery of the situation. This approach generally does not provide sufficient flexibility to take account of local specificities neither sufficient involvement of local populations in the definition of these programs.

\subsection{Ensuring the radiological monitoring}

Post-accident management experience from Chernobyl and Fukushima has pointed out the crucial role of the participation of the residents to the radiological characterization of the territories for people living in affected areas (Ando, 2018).

For assessing external exposure of people living in affected territories, several experiences of local communities have emphasized the need to go beyond the measurements of dose 


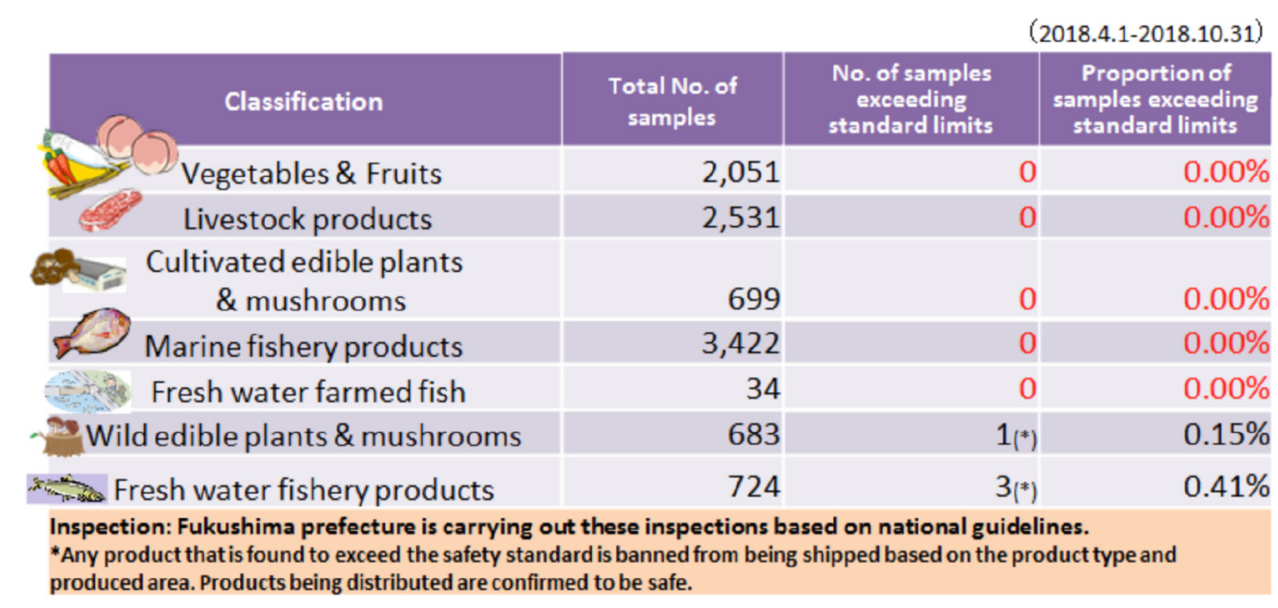

Figure 3. Foodstuff monitoring results. Synthesis of the analyses carried out within the Fukushima Prefecture from April 1st to October 31 st, 2018 (source: Fukushima Prefecture).

rates used to delineate the different zones. For this purpose, the development and diffusion of individual dosimeters in the following years after the Fukushima accident significantly contribute to make objective the situation at stake, taking into account local characteristics as well as habits of each individual. Several publications have shown that actual individual doses could be 4 to 5 times lower than those estimated with the representative dose rates of the areas. (Naito et al., 2016, 2017; ICRP, 2016).

The contamination of food products has in a first phase been a major concern of the population in the affected territories. However, after several years, many people express a progressive disinterest in the monitoring of food products. As soon as the radiological situation is characterized, if the results of measurements show a low contamination of the food products (Fig. 3), the concern and the vigilance are then likely to decrease.

Facing this situation, there is therefore a real challenge to put in place with local communities, actions that arouse their attractiveness. It is essential, for radiological protection experts, to avoid relying solely on scientific considerations to deepen knowledge about the evolution of the radiological situation in the environment. In this context, actions are being taken to set up new cooperation frameworks involving local population and local authorities with the support of experts and universities such as in Kawauchi, Suetsugi or Yamakiya.

Another issue concerns the large program of decontamination set up in the following years after the accident. This program reflects a willingness to recover and to clean as much as possible the affected territories. One drawback of this program is notably the large production of waste accumulated in the temporary storage sites located in the villages ${ }^{6}$, with a variety of contamination levels but with a large part with low radiological concentration (under $8000 \mathrm{~Bq} / \mathrm{kg}$ ). Currently, the authorities are developing the waste management strategy to progressively evacuate the waste packages stored in each

\footnotetext{
${ }^{6}$ Japanese Ministry of the Environment. Environmental Remediation in Affected Areas in Japan, May, 2019. http://josen.env.go.jp/en/pdf/ environmental_remediation_1905.pdf.
}

municipality to various waste storages and disposals depending on the radiological concentration. There is still a significant concern for local population on the transport and presence of radioactive waste in their local environment, mainly leading to a landscape degradation.

The radiological contamination of forests also remains a concern for many residents. In some areas, the forests are still inaccessible while in other areas, it is mainly the picking of some types of mushrooms and wild plants (sansai) that is prohibited. Several actions have been launched to evaluate the doses received during stays in the forest areas for recreational activities and the radiological monitoring of the products collected in the forests is carried out regularly in many municipalities, for example in Kawauchi, Iitate, Suetsugi, Yamakiya. For a large part of the population, rehabilitating the forest areas is a challenge that needs to be addressed. Even though several research institutes are engaged in decontamination experiments on forest areas, this decontamination strategy represents a real challenge while questioning the relevance and the capacity to undertake such decontamination actions.

\subsection{Developing public health program}

Shortly after the accident, the Prefecture set up the 'Fukushima Health Management Survey' including a "basic survey" (behavior during the following months after the accident), a thyroid ultrasound examination, a complete health check of former residents, a survey on the psychological state as well as the lifestyle adopted and finally, a survey dedicated to pregnant women and their newborns (Kumagai and Tanigawa, 2018). In addition, mobile devices for whole body contamination monitoring were set up in response to the concerns of the populations affected by the accident (Hayano et al., 2015).

Several years after the Fukushima accident, there are still strong public concerns of local populations about the potential health consequences of living in the contaminated territories, especially for their children. To improve the interaction with local populations, diverse forms of dialogue and communication actions with various groups of populations have been progressively developed. The training of health professionals, 
notably Public Health Nurses, has been put in place (Goto et al., 2018). Several radiological protection experts have also been involved in the training of staff from local municipalities, to support them to interact with local people living in contaminated territories and to favor the implementation of public health programs addressing the consequences of the accident.

More broadly, the modifications of the demography of local communities and of their daily life after the Fukushima accident call for an evolution/adaptation of the healthcare system itself. Besides the traditional role of the healthcare system, new roles are devoted to health professionals following the nuclear accident (Nishikawa et al., 2016). Among them, there is clearly an increasing role on social and psychological support to be provided to local communities as well as to accompany the development of the radiological monitoring at the service of local people including the promotion of places of dialogue. In addition, the increasing proportion of elderly people in local communities and the decrease of local populations in some areas imply to adapt the health infrastructure. However, there is a strong commitment of local and regional authorities to reinforce the health infrastructure dedicated to people living in affected areas and to anticipate the possible evolution of the areas. Finally, health effects have been observed associated with changes in the life style of affected populations due to significant social, economic and psychological disturbances induced by the accident (Hasegawa et al., 2016). In response to this situation, there is a need for adapting the healthcare system to better address the general well-being of local communities living in affected territories.

\section{The role of radiological protection experts}

As mentioned in the previous part, populations living in territories affected by the Fukushima accident are facing various issues as, among others, the radiological situation of their environment and its possible impacts on human health. Shortly after the Fukushima accident, in a climate of mistrust towards the authorities and official institutions, some communities decided to take initiatives, in order to better understand their situation and build progressively their own references. These actions took different forms, as for instance the mapping of the local contamination, the monitoring of internal and external exposures, decontamination works or even the monitoring of local foodstuff. Therefore, all these initiatives have allowed local people to 'make radioactivity visible' and to better grasp the issues related to their environment. It should be highlighted that, while authorities have been left out of these initiatives, radiological protection professionals and experts in radiological protection have been invited by local people to support them, notably for doing measurements and analyse the results, as well as answering to their questions and worries. In this way, some professionals and experts have gradually engaged themselves voluntarily at the service of the population in the so-called coexpertise processes.

\subsection{The co-expertise process in post-accident situations and the key role of radiological protection experts}

As it was already the case following the Chernobyl accident (Lochard, 2013), feedback experiences from the
Fukushima accident clearly pointed out that radiological protection experts can play a key role for the empowerment of affected people through the co-expertise process. It appears that the role of these experts can take various aspects, beginning with the joint characterisation of the radiological situation with the local populations. However, it should be mentioned that, as experts are already aware about the radiological situation, the first challenge for them is to learn to open their mind to the concerns and worries, but also the expectations expressed by local populations focused on both the day to day life and the possible future. From there, experts can define, jointly with the population, a radiological monitoring which corresponds to local needs and specificities. This challenge is the heart of the co-expertise process, namely: considering not only the scientific knowledge, but also the contribution of local populations who can provide accurate information about their local environment and their habits. On this basis, experts can also play an important role to identify, with the help of local populations, possible protective actions to improve the current situation, whether at individual or community levels, as well as to allow people to take decision about their future in a more informed way. All along the coexpertise process, experts are also involved in helping local people to better understand the various issues related to radiological protection. Therefore, for the experts, the challenge consists in identifying practical and accurate information, which will be useful for the residents in their daily life. In fact, the aim is not to promote a scientific and technical knowledge, but rather to provide practical advices in radiological protection which will help people to regain control of their situation and to take informed decision (Takamura et al., 2016).

Furthermore, it is important to have in mind that radiological protection experts' work not only relies on their own efforts but also on public authorities' ones, notably from the municipalities. Indeed, in the case of co-expertise processes, radiological protection experts can play a key role to relay the expectations and priorities of local population directly to the local authorities which can therefore adapt their strategies. However, radiological protection experts can also be involved through the local authorities themselves. From there, the expected role of radiological protection experts is to support authorities' needs regarding for instance the development of a practical radiological protection culture, or the practical implementation of decontamination or public health programs (Murakami et al., 2017). In this particular case, experts bring their scientific knowledge while local authorities bring the local knowledge about health and environmental status as well as the direct contact with their citizens.

Moreover, and as mentioned before, the post-accident situation affects all dimensions of daily life and so, local population is clearly not only facing issues related to 'radioactivity'. In this context, experts should develop and implement strategies at the service of improving living conditions as a whole. Once again, this is a real challenge for them, as they have to open up and consider many complex issues sometimes out of the scope of their own professional skills. For instance, some inhabitants' concerns can deal with health issues, loss of their job, separation from their family members, discrimination and stigmatisation from the others, difficult access to healthcare, etc. For all these issues, experts 
cannot claim to have all the answers. They need to develop a multidisciplinary approach, call on other experts (psychologists, economists, medical doctors, etc.) and extend the coexpertise process to them. The experts also need to commit themselves for a long-time.

Nevertheless, post-Chernobyl and post-Fukushima situations have clearly shown that, in many cases, co-expertise processes are successful and allow local people to cope with their situation (Ando, 2018). In addition to acquiring a practical radiological protection culture, local populations also succeed to take control of their daily life, and so, regain autonomy to make their own choices in a more informed way. In other words, it could be said that co-expertise processes propose a way to restore dignity of populations living in contaminated territories. And that is why it is important to highlight that the personal engagement of experts in coexpertise processes is essential, although some ethical issues can be raised.

\subsection{Ethical challenges that are facing radiological protection experts}

As seen before, the empowerment of populations living in contaminated territories, thanks to the co-expertise process, is a key element to improve their well-being. However, the role played by the experts in these initiatives could be subject to some questions and even criticisms. Indeed, the empowerment of local populations could be interpreted by some as a strategy to let them manage their situation alone, with no support from the public authorities. Moreover, the close relationship between experts and local populations could also be seen as a risk of manipulation by experts, forcing people to live in contaminated territories and ensuring them that everything is safe. Therefore, to overcome this criticism, experts should respect and behave according to some ethical values (ICRP, 2018; Lochard, 2016; Oughton, 2016).

First, experts have to respect the ethical value of beneficience/non-maleficence. Their role is indeed to organize a long-term vigilance of the territory and to ensure the environmental and health surveillance of the populations. Experts have also the duty to improve the well-being of the population, by notably considering all dimensions of wellbeing, and not only the radiological aspect.

Another challenge for the experts is to respect the dignity of individuals living in contaminated territories, allowing them to make informed decisions. To that extend, experts have to put aside their own convictions to accompany people and help them to make their opinion, without influencing them. However, it should be noted that this autonomy does not correspond in any way to an isolation of the individuals. On the contrary, it stresses the importance of having a balanced relationship between the concerned parties, notably experts, local populations and public authorities.

Experts have also to take actions according to justice and equity between individuals and local communities. Indeed, experiences from Chernobyl and Fukushima have shown that situations and expectations can vary from one community to another, and radiological protection issues can be quite different. Thus, this is the role of experts to adapt their expertise to each local specificity and individual's need, without any preference or special privilege. On this basis, experts have to promote fair access to measurements, monitoring devices and information on the situation. However, this does not mean that experts should highly encourage each person to take measurements or to question their environment. Experts should of course respect the choice of those who do not wish to be involved in the co-expertise process.

The notion of transparency is also important insofar as any co-expertise process requires the respect of rules of cooperation between the various stakeholders. Sharing of measurement results and their analysis to all members of the community is a key point on which experts have to contribute.

One of the ethical challenges is to ensure sufficient protective measures be implemented by authorities to avoid unacceptable individual risk taking into account the remaining uncertainties on the effects of radiation at low doses. Therefore, radiological protection experts have to support the optimisation principle aiming to maintain or reduce exposures as low as reasonably achievable, considering the socio-economic aspects (ethical value of prudence as well as consideration on accountability).

Before concluding this part, it should be highlighted that uncertainties underlie all the assessment and the management of radiological risk. In this sense, experts have to acknowledge the difficulty to manage post-accident situations. Their role still remains to provide protection and to avoid any unacceptable risks for local populations; this is their accountability.

\subsection{The embodied values of radiological protection experts}

The analysis of co-expertise processes implemented in contaminated territories following Chernobyl or Fukushima accidents show that, in addition to respecting the ethical values mentioned above, experts often adopt a particular posture, dialogue with affected populations requiring experts to demonstrate some human qualities.

Indeed, experts involved in co-expertise processes often have a certain capacity of listening and receiving what the local populations want to share with them. In this way, experts can better understand the local needs and concerns, and so develop the most appropriate and accurate protective actions with them. The role of experts, as we have seen, is not to force countermeasures on the inhabitants, but on the contrary, to respect their choices and to share their doubts. In that sense, experts involved in co-expertise processes have to be empathetic, and to know how to put themselves 'at the service of' and certainly not 'in place of'.

Moreover, the situation lived by the affected populations calls on very different issues, within which the radioactivity is not the single one. Experts have to acknowledge the complexity of this situation. They are not able to provide all the answers to the populations' expectations, and in that respect, experts have to be humble and accept to call on other experts.

Likewise, experts have to show humility by acknowledging the fact that they will never fully understand, as well as the local populations do, the impact that may have a nuclear accident on the daily life. In that sense, experts should not 
place themselves as those who know what to do to protect people and to improve their situation. Their role is rather to recognize that the radiological contamination is not legitimate in their territory, and that the rehabilitation process will be long, tedious and cannot be done without the help of local populations. In other words, they should accept that their duty is not to work for the population but to work with the population.

Finally, it should be highlighted that experts are both involved with public authorities, local populations and other stakeholders. They can be facilitator between these two, and so, try to promote and coordinate actions implemented at local, regional and national levels. As experts involved in coexpertise processes are fully aware of the difficulties faced by local populations, they can also be a relay with the national and international scenes, particularly to testify of the situation.

Feedbacks from post-Chernobyl and post-Fukushima situations show that, after many years of involvement with local populations, experts have developed personal relationship with them. Indeed, very often, experts continue to engage themselves in contaminated territories during their personal time when they are no longer representing their institutes. This raises some questions about the sustainability of these coexpertise processes. Also, it should be noted that involvement with local community takes time. Therefore, it is not manageable for the experts to duplicate as such their work with all the other communities. A question can then be raised: how to ensure the dissemination of these co-expertise processes to other communities? These are examples of challenges that Japanese communities are dealing with nowadays, and which are further detailed on the last part of this paper.

\section{Main challenges for the radiological protection experts}

The Fukushima experience, like the Chernobyl one, confirmed that radiological protection experts are generally not prepared to deal with the complex consequences of nuclear accidents. These difficulties, which appear from the accident phase, are still present today, as people living in contaminated territories are still confronted with complex issues in their daily life and so need to be supported by experts in the long term. Today, several years after the Fukushima accident, it turns out that experts are facing two main challenges in Japan:

- the diffusion of the co-expertise processes, which need to be extended to local communities which are not involved yet;

- and the sustainability of vigilance in the contaminated territories.

\subsection{Disseminating the co-expertise processes with local communities}

Experiences from post-accident management in Chernobyl and Fukushima highlight some difficulties in spreading initiatives taken by some local communities to others, which are facing similar situations. It turns out that, while local communities involved in co-expertise processes can promote these processes and share their results with other communities, they generally do not have the legitimacy to support and initiate new co-expertise in other territories.

For their part, radiological protection experts could play this role, and so, try to initiate dialogue and sharing between communities in order to help involvement of communities, which have not started a co-expertise process yet. However, it should be noted that the number of experts involved in such coexpertise approaches remains quite limited.

Among the experts involved in the initiatives presented in this paper, it should be noted the low involvement of experts from public expert bodies, while experts from Universities, health professionals as well as some citizens are more involved. Among the reasons for the limited involvement of experts in such processes, one can notice:

- loss of confidence in organizations and institutional experts following the accident;

- type of activities rarely registered in the missions of institutional organizations;

- lack of culture regarding the dialogue with stakeholders for all experts;

- apprehension of having to intervene in a situation in which technical expertise is only one component.

There is no need for deep expertise in social sciences to be able to intervene in these contexts but rather an awareness of the human dimensions of the post-accident situation and the willingness to open the dialogue and share views and expertise with the stakeholders.

In addition, it is important to point out that co-expertise processes can not be duplicated from one community to another, they should rather be adapted to the local specificities and the populations' needs so that it could have real added value for them. On this basis, some local initiatives can be highlighted:

- Nagasaki University, for example, has adapted the model developed for the Kawauchi community to the Tomioka community, whose evacuation order has been lifted in April 2017. Therefore, actions provided to the Kawauchi residents have been also implemented for the Tomioka residents, adapting it accordingly with the local specificities (elderly, isolated population, etc.). Nowadays, experts from the Nagasaki University divide their time among Kawauchi and Tomioka actions;

- the ICRP dialogue initiatives, which are organised in various communities affected by the Fukushima accident, are also a way to share local initiatives and arouse interest in new communities (Lochard et al., 2019).

Analysis of the Fukushima situation also shows that it is important to encourage the implementation of coordination structures, notably to allow a better sharing between experts and communities. These kinds of network would be the occasion to analyze and identify the strengths and weaknesses of each co-expertise approaches, and so try to improve them. These networks could also lead to share common tools, means and efforts, while respecting autonomy to each expert and community. In Japan, the implementation of such networks still needs to be further developed. However, it should be noted that, in the Fukushima Prefecture, some initiatives are 
proposed by public authorities, as for example, the 'counselors network' coordinated by the 'Support Team', which aims to gather all counselors who are in charge to support local population with the radiological monitoring. Indeed, this network allows counselors to share their daily activities, try to solve some difficulties that they are facing, etc. However, it should be noted that this kind of network is focused on a single issue: the radiological support, while many other issues are at stake in contaminated territories. Networks proposing multidisciplinary approaches, and so gathering experts from various background (sociology, economy, medicine, psychology, etc.) should be highly encouraged.

So, today, several years after the accident, one of the main challenges for the radiological protection experts is to accompany the emergence of local coordination structures which could stimulate the involvement of new communities, as well as convince new experts to engage themselves in coexpertise processes.

\subsection{Ensuring the sustainability of the approach}

Several years after the accident, the question of maintaining vigilance arises with regard to the sustainability of local initiatives. This issue has emerged similarly in initiatives developed in post-accident management in Belarus or Norway after the Chernobyl accident. Since the local populations have been involved in a co-expertise process and have gradually taken up the stakes of the local radiological situation, it is necessary to identify the modalities for maintaining vigilance over the long term. It is not possible for local populations to remain mobilized on radiological characterization once the measurements have been made. However, the maintenance of vigilance appears necessary. On the one hand, the vigilance will ensure that the protective strategies put in place to maintain or reduce exposures are still effective and adapted to the evolution of the environment and the socio-economic situation. On the other hand, it will contribute to the spread of the radiological protection culture to the next generations (Tsubokura et al., 2018).

In this context, it is important to develop approaches that make sense in the long term to mobilize the local populations (identify the measures to be carried out and the frequency of these to avoid the routine, identify the actions contributing to the improvement of the quality of life over time, as well as to the "monitoring" of the environment and the "well-being" of local populations...).

The first level of vigilance is based on the development of a practical radiological protection culture and the maintenance of awareness of what has happened. It is thus necessary to redefine the role of surveillance over time and progressively maintain a minimum of radiological measurements while becoming more involved in transmitting the memory of what happened and has been done. The experience of Chernobyl has shown that transmission of the memory allows local people to maintain their vigilance with a new dynamic and give meaning to their action. This transmission of memory starts with the story-telling of what happened and concerns both the dissemination of actions to other local communities and to national and international communities as well as to younger generations (Duranova and Averin, 2016). In this context, the role of the expert is crucial to favor and support the organization of the vigilance with local communities and to contribute to organize the transmission of the radiological protection culture to the young generations.

Several initiatives can be reported in this perspective:

- the Atlas developed by the community of Suetsugi in interaction with radiological protection experts, providing a new dynamic several years after the accident, contributing to sharing the experience together with mobilizing the villagers to identify the key components for ensuring the sustainability of radiological protection culture in their daily life;

- the involvement of Kawauchi village with Nagasaki University in the dissemination of their experience with the organization of training courses in their community and the testimonies provided regularly by the residents;

- the network of citizens set up to provide support to the farmers of Yamakiya, initiated by radiological protection experts.

Involving local communities in post-accident management research at the regional, national and international levels also contributes to maintain the vigilance on radiological protection issues in the daily life. In providing testimonies on their experience in research projects or workshops, local communities have to regularly review the current situation and to evaluate the implementation of protection strategies. Experts play a key role in this domain. This clearly shows the role of approaches that do not leave the local population alone in the organization of vigilance, requiring shared responsibility.

The second level deals with the socio-economic development of the territories. The feedback from Chernobyl postaccident management and the analysis of the current situation in Fukushima Prefecture underline the importance of integrating the vigilance on the radiological situation in socio-economic projects supporting the development of the territory. In fact, organizing the vigilance without ensuring the socio-economic development of the territory has little chance of lasting. For local populations, vigilance cannot be a project in itself. Existence of local projects help to give meaning to the lives of people in the territories and enable them to look to the future. It is in this context that vigilance makes sense and can contribute to the sustainability of the socio-economic development ensuring due consideration to the radiological context and providing the capacity for the local population to maintain or reduce exposures as low as reasonably achievable in a sustainable manner taking into account the specific context.

In this perspective, the role of radiological protection experts focuses on the development, evaluation and support of the implementation of these territorial projects to consider the local radiological situation and its evolution. This role implies an interaction with local populations, local and national authorities as well as possible other stakeholders. This raises the question of putting in place mechanisms, involving stakeholders from different origins and allowing the emergence and support of territorial projects taking into account the radiological situation. The sustainability of these projects largely relies on the allocation of sufficient and sustainable resources dedicated to support the activities jointly developed 
by local stakeholders and local authorities with radiological protection experts, and addressing together a wide range of issues of health and social life.

\section{Conclusion}

One of the main features concerning the role of radiological protection experts in the recovery of post-accident situation is to put the co-expertise process at the service of improving the living conditions of the affected communities, contributing to the development of an informed decision process for the residents facing the situation. Among the challenges to be dealt by radiological protection experts, there is the need for establishing the conditions and means for developing a shared representation of the radiological situation between experts and the affected residents as well as an ethical challenge regarding the position to adopt to ensure the fairness of the engagement process respecting autonomy and justice for the different populations affected.

In the recovery phase, the radiological protection issues integrating health and environmental protection have to be considered in a broader context where the main challenges are related to the decent conditions of life and a sustainable socioeconomic development in the affected territories. For the experts, engaging a pluri-disciplinary approach is essential and calls for setting up cooperation processes with local stakeholders and other experts from other disciplines and other origins to address the key challenges notably the role of the radiological monitoring, public health issues, socio-economic issues, environmental management... The sustainability of the life in the territories is at stake and radiological protection issues need to be maintained, requiring for the experts to find their place in the long-term.

As discussed above, the traditional role of expert assessing the situation and providing technical and scientific advices for the improvement of the protection is no more sufficient in the recovery situation. To implement the coexpertise process with local stakeholders, experts and their organizations need to revisit their skills and management. It is important to be aware of the particular needs and expectations of the affected people as well as challenges beyond the radiological protection issues. It is also important to be engaged on a step-by-step process, which evolves based on various local, national, international factors and with time. Having no unique approach, flexibility is required both in terms of mobilization of expertise as well as in terms of means provided and process developed to accompany the local initiatives. The mandate provided by the organizations to their experts has to be adapted to the specific situation and adequate support to the experts themselves has to be considered. It is essential to organize an adequate sharing of responsibilities and develop multilevel engagement process of institutions and stakeholders including an evaluation process for ensuring the implementation of an efficient strategy.

\footnotetext{
${ }^{7}$ European research projects CONFIDENCE and TERRITORIES, ICRP Task Group on update of publications 109 and 111, Expert Group on Recovery Management at the Nuclear E.
}

Sharing experience from post-accident management on the role of expert in involving local populations contributes to improving the preparedness and developing a framework to deal with recovery issues. Several European and international projects $^{7}$ are currently addressing this issue. Engaging cooperation and developing education and training activities with experts and organizations already involved in recovery management are essential for really addressing the new role of radiological protection expert in the development of coexpertise processes at the service of local stakeholders.

\section{Acknowledgments}

This paper benefited from the main lessons of the Fukushima Dialogue meetings initiated by the International Commission on Radiological Protection (ICRP). Analyses partly funded by the TERRITORIES project (as part of the CONCERT project) have been also considered for this paper. TERRITORIES project has received funding from the Euratom research and training program 2014-2018 under grant agreement No.662287. This publication reflects only the author's view. Responsibility for the information and views expressed therein lies entirely with the authors. The European Commission is not responsible for any use that may be made of the information it contains.

\section{References}

Ando R. 2016. Measuring, discussing, and living together: lessons from 4 years in Suetsugi. Ann ICRP 45(1S): 75-83.

Ando R. 2018. Trust-what connects science to daily life. Health Physics 115(5): 581-589.

Ban N. 2016. Japanese experience in stakeholder involvement: ICRP dialogue meetings. Radioprotection 51(HS1): S51-S53.

Baudé S, Hériard-Dubreuil G, Schneider T. 2016. Local populations facing long-term consequences of nuclear accidents: lessons learnt from Chernobyl and Fukushima. Radioprotection 51(HS2): S155-S158.

Chiyoda Technol. 2018. Specifications of D-shuttle. Available from www.c-technol.co.jp/eng/e-dshuttle.

Duranova T, Averin V. 2016. The value of exchange visits. Radioprotection 51(HS1): S47-S49.

Gariel JC, Rollinger F, Schneider T. 2018. The role of experts in postaccident recovery: lessons learnt from chernobyl and Fukushima. Proceedings of the Fourth International Symposium on the System of Radiological Protection. Ann ICRP 47: 3/4.

Goto A, Lai AY, Kumagai A, Koizumi S, Yoshida K, Yamawaki K, Rudd RE. 2018. Collaborative processes of developing a health literacy toolkit: a case from Fukushima after the nuclear accident. Journal of Health Communication 23(2): 200-206.

Hasegawa A, Ohira T, Maeda M, Yasumura S, Tanigawa K. 2016. Emergency responses and health consequences after the Fukushima accident, evacuation and relocation. Clinical Oncology 28(4): 237-244.

Hayano R, Tsubokura M, Miyazaki M, Ozaki A, Shimada Y, Kambe T, Nemoto T, Oikawa T, Kanazawa Y, Nihei M, Sakuma Y, Shimmura H, Akiyama J, Tokiwa M. 2015. Whole-body counter surveys of over 2700 babies and small children in and around Fukushima Prefecture 33 to 49 months after the Fukushima Daiichi NPP accident. Proceedings of the Japan Academy, Series B, Physical and biological sciences 91(8): 440-446. 
ICRP. 2016. Proceedings of the International Workshop on the Fukushima Dialogue Initiative. Ann ICRP 45(2S).

ICRP Publication 138. 2018. Ethical foundations of the system of radiological protection. Ann ICRP 47(1).

Kotoba. 2015. Dialogues in Fukushima. The story of four years of dialogue for the rehabilitation of living conditions in the areas contaminated by the Fukushima accident, Web documentary, available from http://www.fukushima-dialogues.com.

Kumagai A, Tanigawa K. 2018. Current status of the Fukushima Health Management Survey. Radiation Protection Dosimetry 182 (1): 31-39.

Kurihara M, Onda Y, Kato H, Loffredo N, Yasutaka T, Coppin F. 2018a. Radiocesium migration in the litter layer of different forest types in Fukushima, Japan. Journal of environmental radioactivity 187: 81-89.

Kurihara M, Onda Y, Suzuki H, Iwasaki Y, Yasutaka T. 2018b. Spatial and temporal variation in vertical migration of dissolved $137 \mathrm{Cs}$ passed through the litter layer in Fukushima forests. Journal of environmental radioactivity 192: 1-9.

Kuroda Y, Iwasa H, Orui M, Moriyama N, Suemoto CK, Yashiro C, Matsuda K, Yasumura S. 2018a. Risk factor for incident functional disability and the effect of a preventive exercise program: a 4-year prospective cohort study of older survivors from the Great East Japan earthquake and nuclear disaster. International Journal of environmental Research and Public Health 15(7): 1430.

Kuroda Y, Iwasa H, Orui M, Moriyama N, Nakayama C, Yasumura S. 2018b. Association between health literacy and radiation anxiety among residents after a nuclear accident: comparison between evacuated and non-evacuated areas. International Journal of environmental Research and Public Health 15(7): 1463.

Liland A, Skuterud L. 2013. Lessons learned from the chernobyl accident in Norway. Social and ethical aspects of radiation risk management. Radioactivity in the Environment 19: $157-176$

Lochard J. 2013. Stakeholder engagement in regaining decent living conditions after chernobyl. In: Social and ethical aspects of radiation risk management (D. Oughton, S.O. Hansson, Eds.). Radioactivity in the Environment 9: 311-331.

Lochard J. 2016. First Thomas S. Tenforde topical lecture: the ethics of radiological protection. Health Physics 110: 201-210.

Lochard J, Schneider T, Ando R, Niwa O, Clement C, Lecomte JF, Tada JI. 2019. An overview of the dialogue meetings initiated by ICRP in Japan after the Fukushima accident. Radioprotection 54 (2): $87-101$
Miyazaki M. 2017. Using and explaining individual dosimetry data: case study of four municipalities in Fukushima. Asia Pacific Journal of Public Health 29(2S): 110S-119S.

Murakami M, Sato A, Matsui S, Goto A, Kumagai A, Tsubokura M, Orita M, Takamura N, Kuroda Y, Ochi S. 2017. Communicating with residents about risks following the Fukushima nuclear accident. Asia Pacific Journal of Public Health 29(2S): $74 \mathrm{~S}-89 \mathrm{~S}$

Naito W, Uesaka M, Kurosawa T, Kuroda Y. 2017. Measuring and assessing individual external doses during the rehabilitation phase in Iitate village after the Fukushima Daiichi nuclear power plant accident. Journal of Radiological Protection 37(3): 606-622.

Naito W, Uesaka M, Yamada C, Kurosawa T, Yasutakat, Ishii H. 2016. Relationship between individual external doses, ambient dose rates and individuals' activity-patterns in affected areas in Fukushima following the Fukushima Daiichi nuclear power plant accident. PloS one 11(8): e0158879.

Nishikawa Y, Tsubokura M, Yamazaki S. 2016. Healthcare delivery to a repopulated village after the Fukushima Nuclear disaster: a case of Kawauchi Village, Fukushima, Japan. Japan Medical Association Journal 59(4): 159-161.

Oughton D. 2016. Societal and ethical aspects of the Fukushima accident. Integrated environmental assessment and management 12(4): 651-653.

Takamura N, Orita M, Taira Y, Fukushima Y, Yamashita S. 2018. Recovery from nuclear disaster in Fukushima: collaboration model. Radiation Protection Dosimetry 182(1): 49-52.

Takamura N, Taira Y, Yoshida K, Nakashima-Hashiguchi K, Orita M, Yamashita S. 2016. Communicating radiation risk to the population of Fukushima. Radiation Protection Dosimetry 1-4.

Tsubokura M, Kitamura Y, Yoshida M. 2018. Post-Fukushima radiation education for Japanese high school students in affected areas and its positive effects on their radiation literacy. Journal of Radiation Research 59(suppl_2): ii65-ii74.

Tsuji H, Yasutaka T, Kawabe Y, Onishi T, Komai T. 2014. Distribution of dissolved and particulate radiocesium concentrations along rivers and the relations between radiocesium concentration and deposition after the nuclear power plant accident in Fukushima. Water research 60: 15-27.

Yasutaka T, Kondoh A, Kobayashi T, Naito W, Kurihara M, Kanai Y, Kanno G, Hirono A, Kanno A, Ohuchi K. 2019. Dialogue about environmental monitoring and some activities with residents in Yamakiya region. What we should do for the future? Proceeding of the international symposium of radiation medical science center of Fukushima Medical University.

Cite this article as: Schneider T, Maître M, Lochard J, Charron S, Lecomte J-F, Ando R, Kanai Y, Kurihara M, Kuroda Y, Miyazaki M, Naito W, Orita M, Takamura N, Tanigawa K, Tsubokura M, Yasutaka T. 2019. The role of radiological protection experts in stakeholder involvement in the recovery phase of post-nuclear accident situations: Some lessons from the Fukushima-Daïchi NPP accident. Radioprotection 54(4): 259-270 